www.jmscr.igmpublication.org

Index Copernicus Value: 79.54

ISSN (e)-2347-176x ISSN (p) 2455-0450

crossrefDOI: https://dx.doi.org/10.18535/jmscr/v7i2.180

Journal Of Medical Science And Clinical Research

IGM Publication

An Official Publication of IGM Publication

\title{
Giant Cell Tumour of Head and Neck of Femur - A Rare Location - Managed with Bipolar-Hemiarthroplasty
}

\author{
Authors \\ Chennakesava Rao Sajja ${ }^{1}$, Satish Chandra Reddy $\mathbf{T}^{2}$, Bhavani Prasad $\mathbf{T}^{3}$ \\ ${ }_{1,2}$ Junior Resident, ${ }^{3}$ Professor \\ ${ }^{1-3}$ Department of Orthopaedics, King George Hospital, Andhra Medical College, Visakhapatnam, India \\ Email:kesav4u6@gmail.com; dr.satishchandrareddy@gmail.com
}

\begin{abstract}
Background: Giant cell tumour of bone is a rare but aggressive benign tumour that typically arises adjacent to the sub-chondral bone of major joints in the epiphyseal area of a skeletally mature patient, mainly around the knee, most often involves distal femur, proximal tibia, proximal humerus, and distal radius , Giant cell tumour of the proximal femur $(P F)$ constitutes only 5.5\% of all giant cell tumours and femoral head and neck is being rare location. Curettage with or without bone cement results in a higher rate of local recurrence and potential morbidity from further surgery. Although it is better to try and preserve the hip joint, particularly in younger patients, this may not be possible, and alternatives such as exicision and hemiarthroplasty, total hip replacement (THR) or proximal femoral endoprosthetic replacement (EPR) may have to be considered.

Materials \& Methodology: 4 young male patients of age between 20-25 years, who have biopsy confirmed Giant cell tumour of femoral head and neck without pathological fracture managed with exision of femoral head replaced with bipolar hemiarthroplasty and followed for $11 / 2$ years in the Department of Orthopaedics AMC/KGH, vizag.

Results: patients were made partial weight bearing on day 1 and made full weight bearing at day 3 after pain tolerated with post operative recovery being uneventful and no reccurance was reported till now with upto $11 / 2$ years follow-up.

Conclusion: Exision and hemiarthroplasty is an better alternative to Curettage with or without bone cement (higher rate of local recurrence) and THR/EPR (which are better options in old age). It preserve acetabular articular surface which is particularly helpful in young patients and also less local recurrence. Keywords: Giant cell tumour, head and neck of femur, bipolar hemiarthroplasty.
\end{abstract}

\section{Introduction}

Giant cell tumour of bone is a rare but aggressive benign tumour that typically arises adjacent to the sub-chondral bone of major joints in the epiphyseal area of a skeletally mature young adults between $20-40$ years, mainly around the knee, most often involves distal femur, proximal tibia, proximal humerus, and distal radius ,Giant cell tumour of the proximal femur(PF) constitutes only $5.5 \%$ of all giant cell tumours and femoral head and neck is being rare location. ${ }^{1}$ As the proximal femur is an uncommon site for this rare 
primary bone tumour, there is little information in the literature about the results of a particular form of treatment at this anatomical site. The treatment options available for such a lesion are intralesional curetting and bone grafting or packing with bone cement; excision arthroplasty (Girdlestone procedure); excision and reconstruction with cortical cancellous bone graft and internal fixation; hemiarthroplasty; total hip replacement (THR) and proximal femoral endoprosthetic replacement (EPR) .Given the nature of presentation of these lesions and the age group affected, the treatment protocol followed for these is expected to give a robust, long standing, permanent, satisfactory outcome with minimal complications. Curettage with or without bone cement results in a higher rate of local recurrence and potential morbidity from further surgery. ${ }^{2}$

The viability of the hip after aggressive curettage can be a real problem, especially in younger patients, despite leaving an intact articular surface. Although it is better to try and preserve the hip joint, particularly in younger patients, this may not be possible, and alternatives such as exicision and hemiarthroplasty, THR or proximal femoral EPR may have to be considered. THR will usually entail only partial excision of the giant cell tumour, with curettage of the remainder, whereas EPR usually facilitates complete removal of all diseased bone and are high cost. Although previous studies have reported reasonable results from treating a giant cell tumour of the proximal femur by replacement (either THR or EPR),no single study to date has looked at other options for treatment and their outcomes. Total hip replacement in a young patient is seen risky due to the high probability of early revision surgery. The purpose of this study was to assess whether the use of bipolar hemiarthoplasty was successful in eradicating giant cell tumours of the proximal femur, or whether an alternative management strategy was more appropriate .We report 4 cases of a 20- 35 year-old patient for whom we opted for hemiartroplasty treatment because of our unfavorable socio-economic context and young age.

\section{Materials \& Methodology}

4 young male patients of age between 20-35 years, who have presented with pain in the groin and lower back between December 2016 and December 2018 in the Department of Orthopaedics AMC/KGH, Visakhapatnam. They had no history of trauma or physical effort and progressive worsening of the pain. At physical examination, the patient presented tenderness on palpation in the anterior aspect hip joint and pain during movement of the hip, with normal range of motion. In all cases the workup included, apart from the routine radiographs, CT and MRI scan of the affected area and routine laboratory investigations.

Radiographs of pelvis with both hips revealed an osteolytic lesion in the head and neck of femur. A CT scan revealed the extent of the lesion which was confined to head and neck with no extension into the surrounding tissues or adjacent bony structures. There was no evidence of any breach of the cortex. Then a needle /open biopsy from anterolateral/lateral approach to establish the histopathological diagnosis. The diagnosis established as benign aggressive Giant cell tumour of femoral head and neck without pathological fracture. Cases with large extra osseous lesion component with no residual bone stock and cases where articular cartilage damage or joint involvement was evident were excluded from this series.

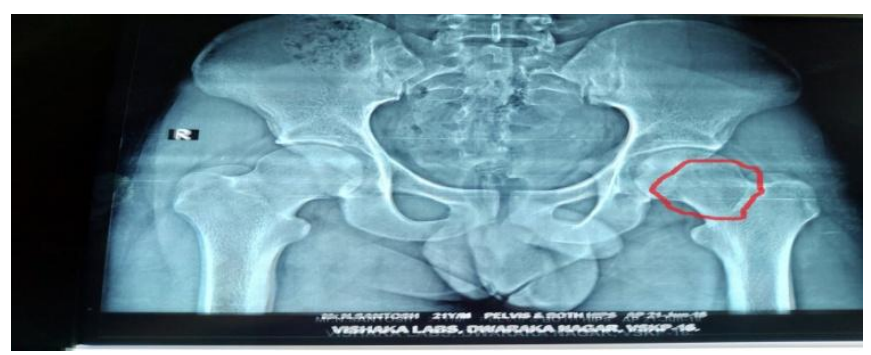

Figure-1: X-ray pelvis with both hips- lytic lesion in left femoral neck with extension into head. 


\section{JMSCR Vol||07||Issue||02||Page 1035-1041||February}

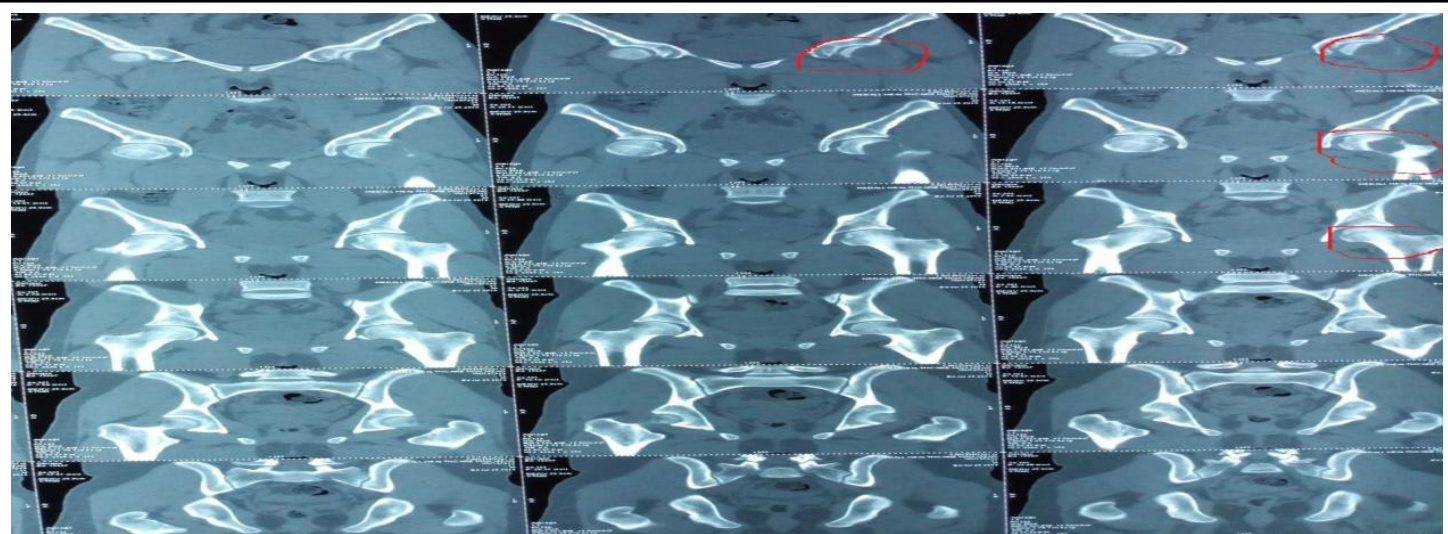

Figure-2: CT SCAN -coronal view -shows Osteolytic/cystic lesion in anterolateral proximal left femoral head and neck.

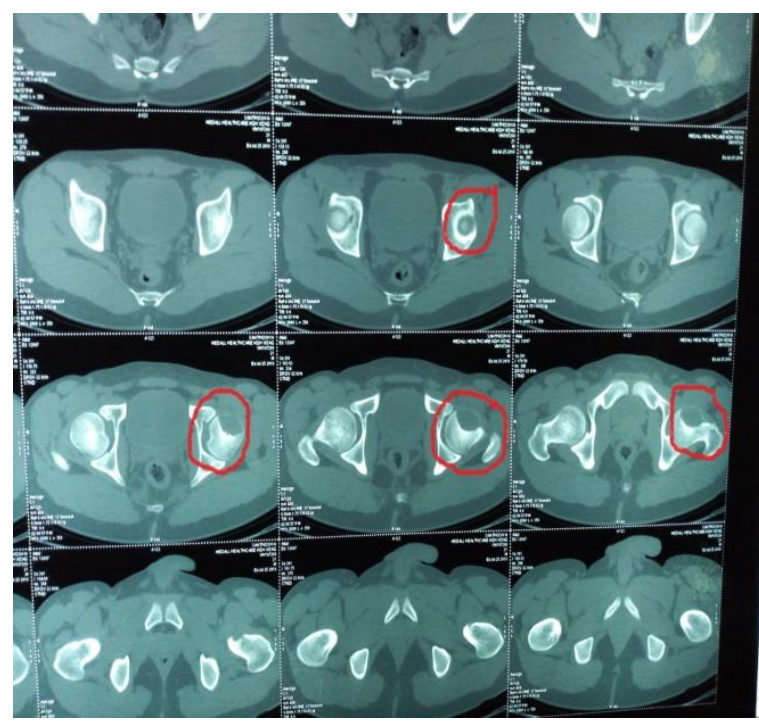

Figure -3 : CT Scan- Axial View in anterolateral proximal left femoral neck size

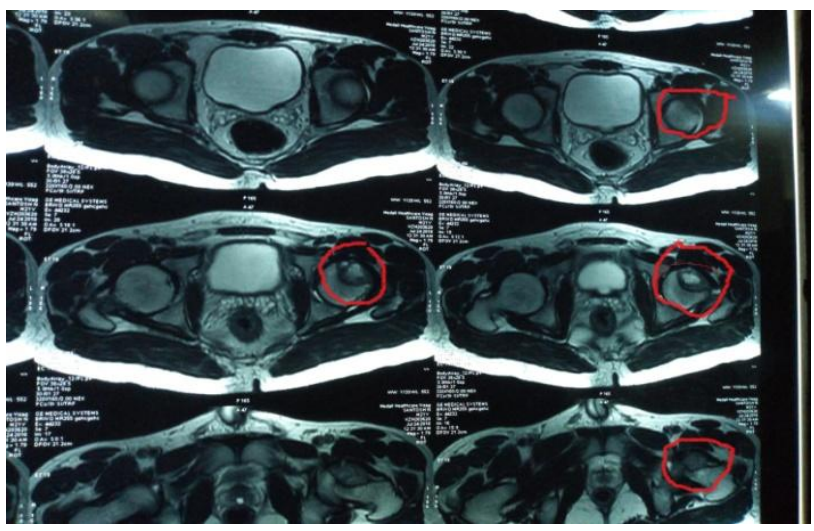

Figure - 4A MRI Both hips axial view

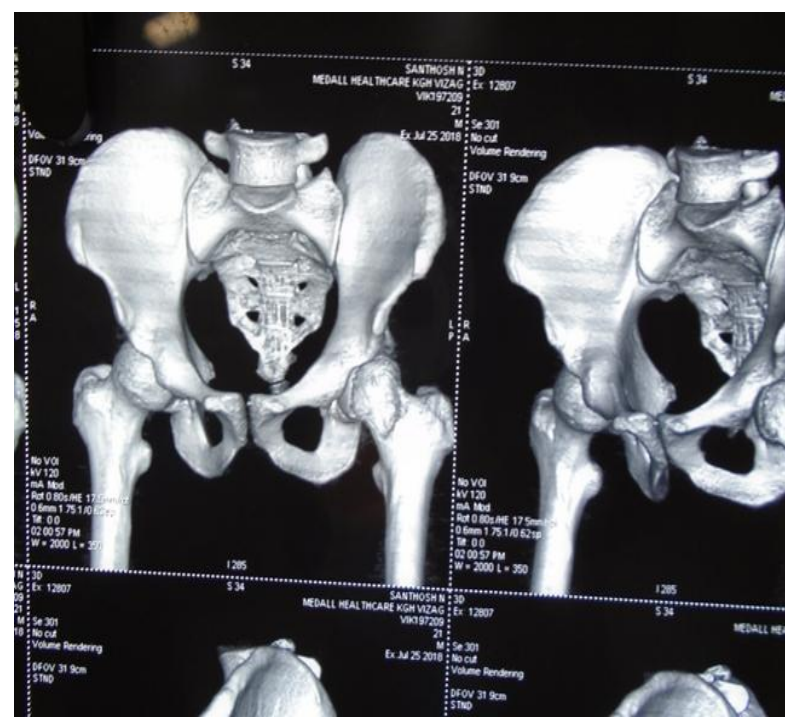

Figure -3B: 3D CT Pelvis -show Osteolytic/cystic lesion

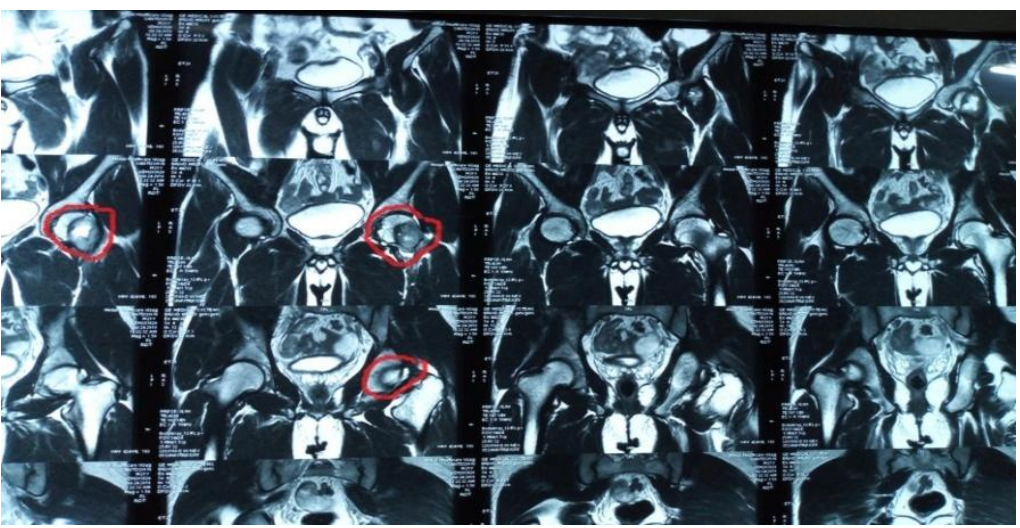

Figure-4B-MRI Both hips -coronal view 


\section{JMSCR Vol||07||Issue||02||Page 1035-1041||February}

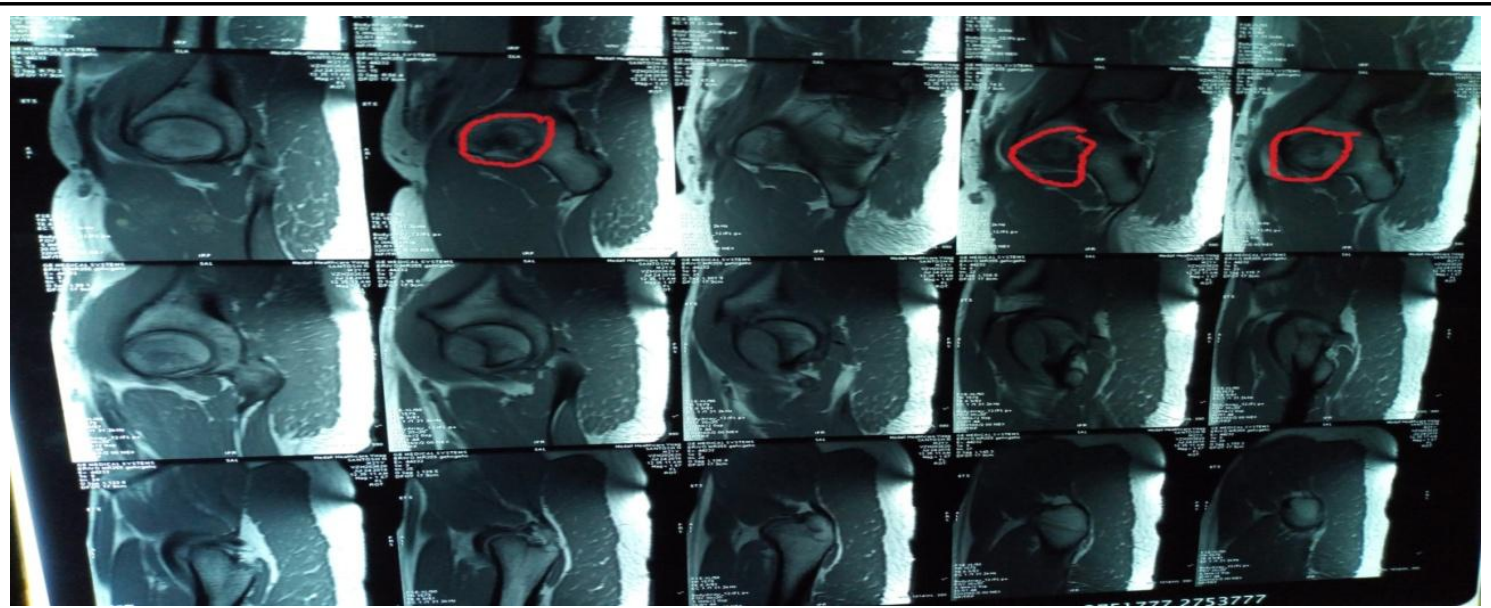

Figure- 4C-MRI -saggital view

Figure 4A, 4B, 4C - MRI pelvis with both hips shows-well defined altered signal intensity lesion involving anteroinferior aspect of the left femoral head and neck with extra-osseous soft tissue limited by joint capsule.

\section{Treatment}

It was decided to try to preserve the acetabulum of joint and attempt to do exision of femoral head to eradicate the tumour and replaced the head with bipolar prosthesis, give the patient a chance to lead a near normal life. We decided not to attempt total hip arthroplasty considering the young age of the patient and the localized nature of the lesion.

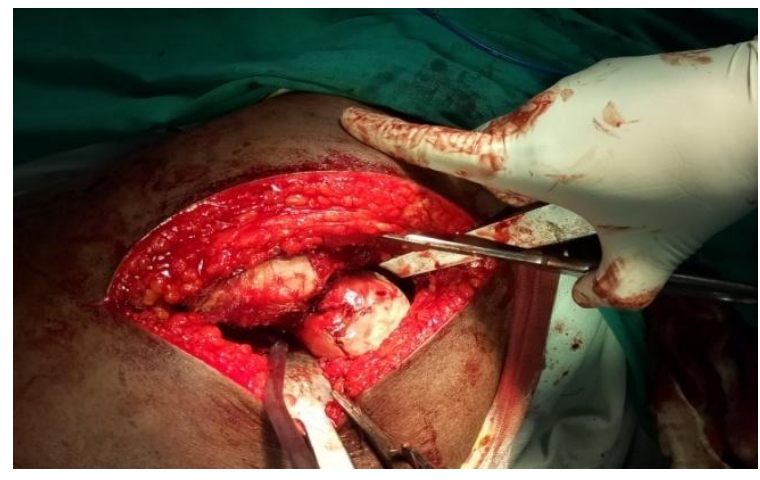

Figure- 5: femoral head dislocation

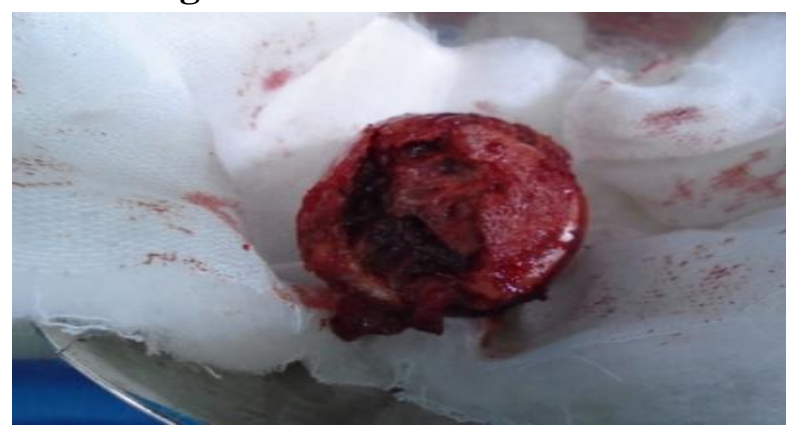

Patient was councelled and explained about GCT characters and its recurrence and management options and future second surgery (like THR) for recurrence.

Surgical Procedure (Routine hemiarthroplasty procedure):

- Spinal anaesthesia

- lateral position.

- Moors approach.

- Exicion of head.

- Bipolar prothesis inserted.

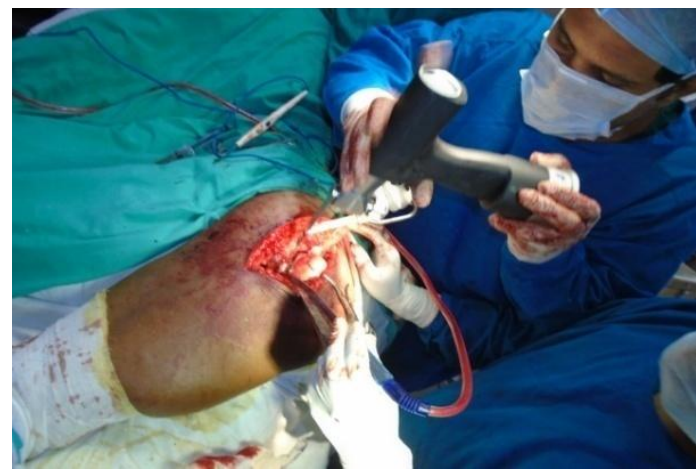

Figure-6-Femoral Neck Cutting

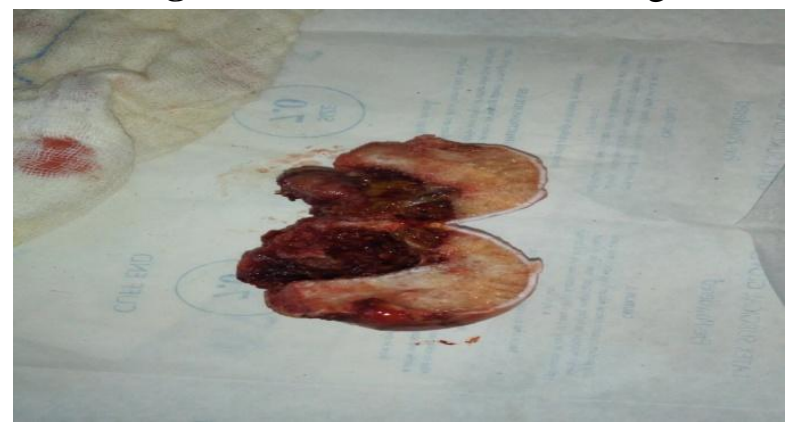

Figure-7: Excised femoral head with cross section 


\section{JMSCR Vol||07||Issue||02||Page 1035-1041||February}

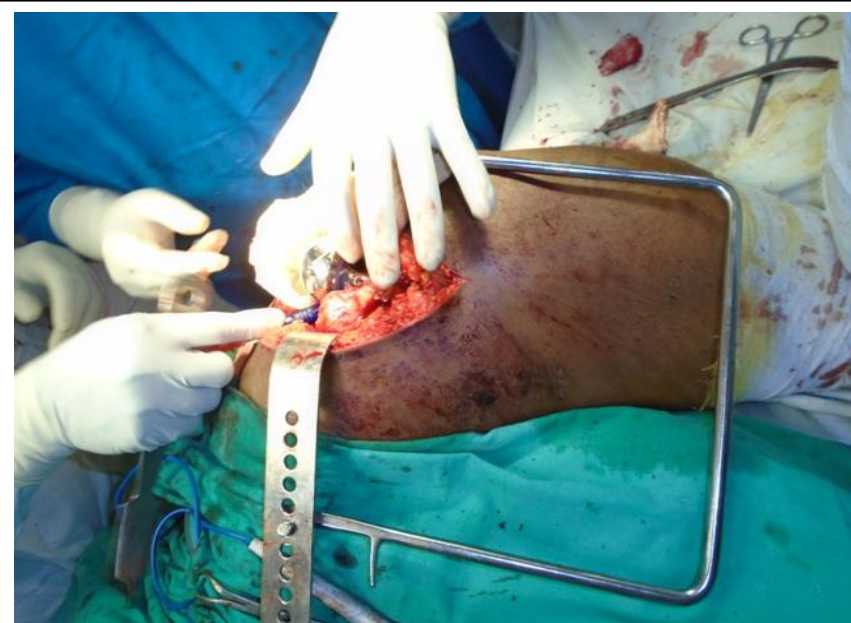

Figure 8A-Bipolar prosthesis inserted

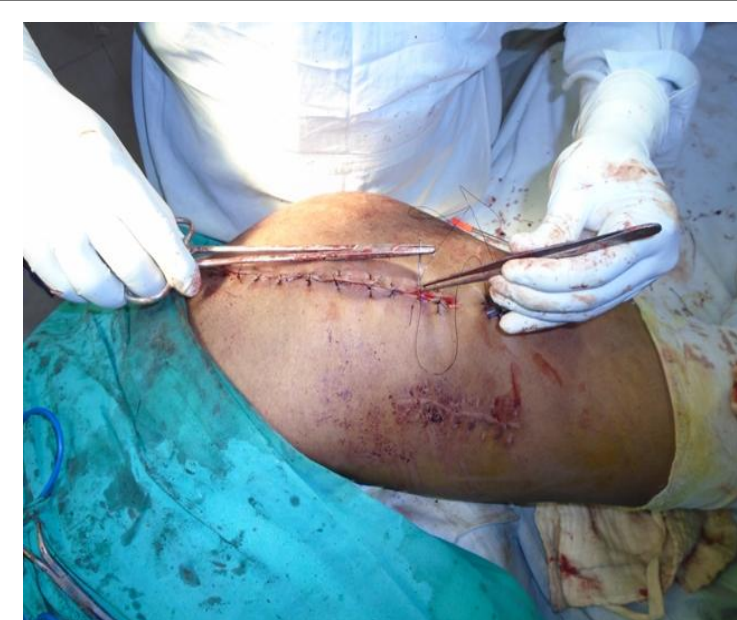

Figure-8B-Closure of surgical wound

Histopathology of the excised head material showed uniform distribution of osteoclast like giant cells in a background of mononuclear cells,

confirming the diagnosis of GCT, and followed up to $1 \frac{1 / 2}{2}$ years in the Department of Orthopaedics AMC/KGH, Visakhapatnam.

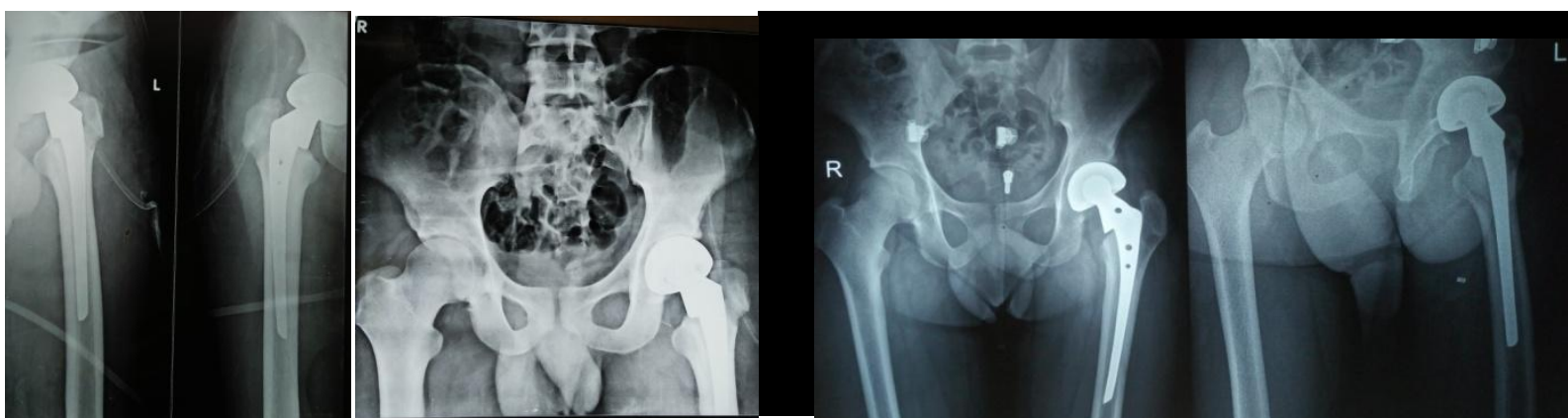

Figure-9A-Immediate post op x-ray
Figure 9B-6 months post op x-ray

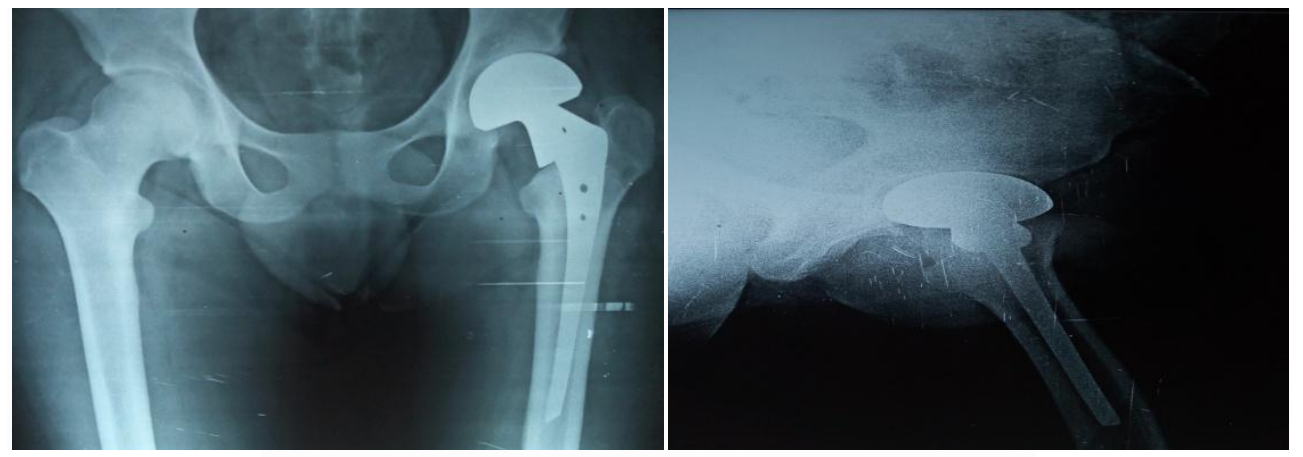

Figure- 10: one year post op follow up x-rays

\section{Results}

Patients were made partial weight bearing on day 1 and made full weight bearing at day 3 after pain tolerated with post operative recovery being uneventful. They were followed regularly in our hospital every 3 months with $\mathrm{x}$ rays with no recurrence of the lesion. At the last follow up $1 \frac{1}{2}$ after surgery, there was no evidence of recurrence locally and patient did not have any pain and was able to do routine daily activities with full weight bearing. The range of movements (flexion, abduction, rotations) was full. There was no limb length discrepancy.

\section{Discussion}

The treatment of GCT is essentially surgical. There is evidence contrary to curettage, but there is a correlation with surgery and method, 
histologic type, tumour size, location, and age of the patient - these are factors that directly influence the prognosis, as well as lesion staging. ${ }^{3}$

The treatment of choice in most GCTs is curettage and bone grafting. Historically, however it has been associated with high rate of recurrence (30\%-50\%) and therefore different adjuvants have been introduced. ${ }^{4}$

Treatments involving substitution, such as reconstruction of the proximal femur with femoral prostheses, the use of proximal endoprosthesis for large replacements of proximal femoral neoplasms, modular titanium endoprosthesis, and conventional total hip arthroplasty are techniques used for GCT of the hip. ${ }^{5-8}$

In giant cell tumours, due to its location in the epiphysis, the GCT often invades the subchondral bone of the joint. Hence, en bloc resection often requires sacrifice of the articular surface and resultant complex reconstruction procedures which can lead to complications, occasional revision operations and decreased quality of life in the long term. ${ }^{9,10}$

Although there may be reluctance to advise hip replacement arthroplasty as the primary treatment of choice in young patients, the likelihood of recurrence following curettage and bone grafting, particularly at this anatomical site, must be stressed, and hemiarthroplasty can be considerd as alternative with low cost and less recurrence rate. It may be that hip replacement arthroplasty should be considered the primary treatment of choice or reserving it as a secondary procedure for recurrences and associated complications if patient affordable as it is high cost procedure.

\section{Conclusion}

Exision and hemiarthroplasty is an better alternative to Curettage with or without bone cement (higher rate of local recurrence) and THR/EPR (which are better options in old age. especially in developing countries like Indian as it is economically effective and high cost procedures like THR and EPR can be reserved for recurrent cases particularly in young patients as revision procedure. It preserve acetabular articular surface which is particularly helpful in young patients and also less local recurrence.

\section{References}

1. Unni KK, Inwards CY, eds. Dahlin's bone tumours. Sixth ed. Philadelphia: Lippincott Williams \& Wilkins, 2010.

2. Gaston CL, Bhumbra R, Watanuki M, et al. Does the addition of cement improve the rate of local recurrence after curettage of giant cell tumours in bone? J Bone Joint Surg [Br] 2011;93-B:1665-1669.

3. Filho RJG, Korukian M, Ishirara HI, Miceno Filho NM,Figueiredo MTB, Seixas MT. É a curetagem um métodoeficiente no tratamento dos tumores ósseos? Rev Bras Ortop.1993;28 (11/12):813-6.5

4. Omar Kirmani, Nasir Muzaffar*, Mohammad Ehsan, Shamim AhmadGiant Cell Tumour of the Femoral Neck: A Rare Site Managed by Curettage and Bone Grafting Only, Global Journal of Surgery, 2013, Vol. 1, No. 3, 22-24.

5. Menendez LR, Ahlmann ER, Kermani C, Gotha H.Endoprosthetic reconstruction for neoplasms of the proximalfemur. Clin Orthop Relat Res. 2006;450:46-51.

6. Croci AT, Camargo OP, Baptista AM, Caiero MT. The use of amodular titanium endoprosthesis in skeletal reconstructions after bone tumor resections: method presentation andanalysis of 37 cases. Rev Hosp Clin Fac Med São Paulo. 2000;55(5):169-76.

7. Donati D, Giacomini S, Gozzi E, Mercuri M. Proximal femurreconstruction by an allograft prosthesis composite. ClinOrthop Relat Res. 2002;(394):192-200.

8. Kulkarni SS, Dogra AS, Bhosale PB. Total hip arthroplasty forgiant cell tumour. J Postgrad Med. 1996;42(3):82-4.

9. O'Donell RKJ, Springfield DS, Motwani HK et al. Recurrence of giant-cell tumors 
of long bones after curettage and packing with cement. J Bone Joint Surg [Am]1994;76-A: 1827-33.

10. Szendroi, M. Giant cell tumour of bone. J Bone Joint Surg Br 86-B (1):5-12, January 2004. 